\title{
EL NUEVO ESCENARIO DE LA POBREZA FRENTE AL COVID-19: ESTUDIO DEL CASO TULANCINGO DE BRAVO, MÉXICO (2016-2021)
}

\author{
O NOVO CENÁRIO DA POBREZA DIANTE DO COVID-19: ESTUDO DE CASO \\ DE TULANCINGO DE BRAVO, MÉXICO (2016-2021)
}

THE NEW COVID-19 POVERTY SCENARIO: TULANCINGO DE BRAVO CASE

STUDY, MEXICO (2016-2027)

Resumen: El programa PROSPERA fue un referente en la lucha contra la pobreza en México durante el período transcurrido entre 2014 y 2019. Según estimaciones de la CEPAL, este Programa de Transferencia Condicionada (PTC) alcanzó una cobertura de $24.13 \%$ de la población en 2018 . El presente artículo tiene como objetivo conocer y analizar el modo como algunos grupos vulnerables han enfrentado la situación actual, esto tras la desaparición de PROSPERA en 2019 y el surgimiento del Covid-19 en febrero del 2020 en México. Esta investigación se enmarca teóricamente dentro de los estudios sobre la pobreza. Se ha realizado un estudio longitudinal que parte de una investigación llevada a cabo en 2016 en el municipio de Tulancingo de Bravo, en la cual se aplicaron entrevistas en profundidad a mujeres beneficiarias de PROSPERA. De tal manera nos hemos dado a la tarea de identificar a algunas de ellas para entrevistarlas nuevamente en 2021. Encontrando que el gobierno federal ha incorporado nuevos programas de atención a personas en condición de pobreza. No obstante, existen escasos apoyos derivados de la situación pandémica. Por otra parte, las mujeres entrevistadas no parecen haber mejorado su condición, además de verse afectadas por la pandemia en múltiples aspectos.

\footnotetext{
' Doctor en Ciencias Políticas y de la Administración y Relaciones Internacionales por la Universidad Complutense de Madrid. E-mail: pabadill@ucm.es
}

Cadernos Prolam/USP-Brazilian Journal of Latin American Studies, v. 20, n. 40, p. 237-262, out. 2027 Edição especial: Trabalho e proteção social na América Latina: desenvolvimentos recentes e os desafios diante da crise pandêmica. ISSN: 1676-6288 
Palabras clave: Pobreza; Programas de Transferencias Condicionadas; Prospera; Pandemia; COVID-19.

Resumo: O programa PROSPERA foi referência na luta contra a pobreza no México durante o período de 2014 a 2019. Segundo estimativas da CEPAL, este programa de transferência condicionada de renda alcançou uma cobertura de $24,13 \%$ da população em 2018. O presente artigo tem por objetivo conhecer e analisar como alguns grupos vulneráveis têm enfrentado a atual situação, depois do término do PROSPERA, em 2019, e do surgimento do Covid-19 no México em fevereiro de 2020. Esta investigação enquadra-se teoricamente nos estudos sobre a pobreza. Foi efetuado um estudo longitudinal a partir de uma investigação realizada em 2016 no município de Tulancingo de Bravo com a aplicação de entrevistas em profundidade com mulheres beneficiárias do PROSPERA. Parte destas mulheres foi entrevistada novamente em 2021. Os resultados apontam para a incorporação de novos programas voltados para indivíduos em situação de pobreza, mas os apoios para enfrentar a pandemia são escassos. As mulheres entrevistadas parecem não ter melhorado a sua condição, além de relatarem que a pandemia as afetou em múltiplos aspectos.

Palavras chave: Pobreza; Programas de Transferência Condicionada de Renda; Prospera; Pandemia, COVID-19.

Abstract: The PROSPERA program was a benchmark in the fight against poverty in Mexico from 2014 to 2019. According to ECLAC, this Conditional Cash Transfer Programme (PTC) reached coverage of $24.13 \%$ of the population in 2018. The main objective of this article is to identify and analyze how some vulnerable groups have faced the current situation, after the termination of PROSPERA in 2019 and the health emergency caused by Covid-19 in February 2020 in Mexico. This article is theoretically underpinned on studies about poverty. Longitudinal research has been carried out based on a study performed in 2016 about poverty in the municipality of Tulancingo de Bravo. In this previous study, we applied in-depth interviews to women beneficiaries of PROSPERA. Part of these women were interviewed again in 2021. After the interviews, we have found that the federal government has incorporated new care programs for poor people. However, there is not enough support to endure the situation caused by the pandemic. On the other hand, the women interviewed do not seem to have overcome their poverty condition and, on top of that, they have been affected by the pandemic in multiple ways.

Keywords: Poverty; Conditional Cash Transfer Programs; Prospera; Pandemic, COVID-19. 


\section{$1 \quad$ Introducción}

De acuerdo a Higgins (2012, p.91), los Programas de Transferencias Condicionadas (PTC) "son transferencias monetarias que se enfocan en las familias pobres y requieren un nivel específico de inversión en capital humano en los hogares de los niños". Uno de los PTC referentes en la lucha contra la pobreza en México fue PROSPERA, esto durante el período (2014-2019). Según estimaciones de la Comisión Económica para América Latina y el Caribe (CEPAL, [s.i.]), este programa tuvo una cobertura de 31,2 millones de personas equivalentes a un $24.13 \%$ de la población en México en 2018.

La presente investigación es un estudio longitudinal que parte de una pesquisa previa realizada en 2016 en el municipio de Tulancingo de Bravo, Hidalgo, México (BADILLO FLORES, 2019). Según datos de CONEVAL (2017, n.p.), para el año 2015 este municipio ocupó la 2a. posición con mayor número de personas en condición de pobreza en el Estado de Hidalgo. De igual forma, la selección fue determinada por ser el lugar de residencia del entrevistador, lo que permitió tener un mejor conocimiento del entorno, de las instituciones y actores de gobierno.

El objetivo principal es conocer y analizar la forma en la que algunas de las mujeres entrevistadas ${ }^{2}$ en 2016 (grupo vulnerable ${ }^{3}$ ) han enfrentado la situación, esto tras la desaparición del programa Prospera y el surgimiento del Covid-19. Lo que nos ha llevado al planteamiento de ciertas

\footnotetext{
${ }^{2}$ De acuerdo al punto 4.1.1, por cada familia beneficiaria se identificaba a una titular, generalmente la madre de familia, quien recibía directamente los apoyos monetarios y debía tener una edad de 15 o más años (SEDESOL, 2015).

3 Según Rodríguez (2001, p. 18), denomina "grupos vulnerables" a "segmentos de la población -como los niños, los ancianos, las mujeres, los indígenas, las jefas de hogar- que, por diversas razones, se consideran en condiciones de indefensión particularmente agudas y que, por lo tanto, requieren de un trato especial de las políticas públicas, lo que origina programas sectoriales y multisectoriales de apoyo y promoción".
} 
interrogantes, por ejemplo, existió algún apoyo de gobierno tras la conclusión de Prospera, con la desaparición del programa cuál ha sido la situación de las mujeres entrevistadas, qué impacto ha tenido la pandemia en su vida laboral, se ha visto afectada la salud de sus familias y si tienen pensado vacunarse. De tal manera, se decidió localizar a algunas de las mujeres entrevistadas en 2016.

La situación pandémica por la que se atraviesa representó un desafío adicional en el trabajo de campo no solo para el entrevistador sino para el entrevistado. Desafortunadamente muy pocas mujeres respondieron, aquellas que lo hicieron mencionaban que el número estaba equivocado, otras directamente se negaban a ser entrevistadas, algunas más preguntaban si era con fines de propaganda electoral e incluso una de ellas se comunicó con una autoridad municipal para reportar la situación. Es comprensible el temor y con ello su negativa a ser entrevistadas, a la fecha del 16 de mayo del 2021 según datos del gobierno de la entidad han fallecido 6.070 personas derivado de complicaciones por Covid-19. No obstante, diez de ellas respondieron favorablemente en un primer momento, de las cuales tres no volvieron a tomar ninguna llamada y una más no se presentó. Por lo que en total pudimos aplicar un total de seis entrevistas en profundidad, las cuales a pesar del número nos han brindado información muy valiosa.

Debido a que las entrevistas fueron cara a cara tomamos las siguientes medidas preventivas: uso de cubrebocas en todo momento, colocación de mascarilla, aplicación de gel antibacterial, mantuvimos una distancia mínima de metro y medio con la persona entrevistada, utilizamos espacios abiertos, además de no realizar más de una entrevista por día. El período de aplicación de las entrevistas en profundidad fue durante el mes de marzo del 2021. Además, protegimos la identidad de las mujeres entrevistadas cambiando su nombre por un sustituto. 


\section{La evolución de la pobreza en el estado de hidalgo: el municipio de Tulancingo de Bravo}

El Estado de Hidalgo cuenta con 84 municipios, entre los cuales tenemos a Tulancingo de Bravo (Mapa). Datos recientes de INEGI (2020, p.166) revelan que su población en 2020 era de 168.369 habitantes, representando el $5.5 \%$ de la población estatal. Según datos del Consejo Nacional de Evaluación de la Política de Desarrollo Social CONEVAL (2020), la entidad se encuentra entre los estados con mayor índice de pobreza en México. En el año 2018, un $43.8 \%$ de la población hidalguense se encontraba en situación de pobreza, esto es equivalente a 1,3 millones de personas (CONEVAL, 2020, p. 14). De igual forma, se señala que Hidalgo contaba con un porcentaje de pobreza de 1,9 puntos porcentuales mayor que el nacional (41. 9\%) (Id.).

Mapa - División municipal del Estado de Hidalgo

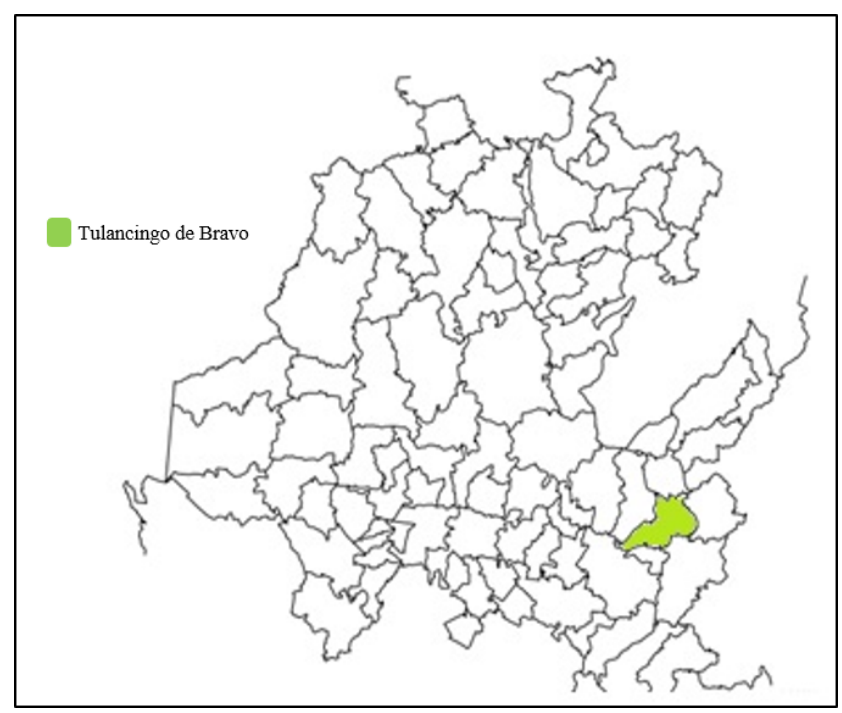

Fuente: elaboración propia a partir de INEGI (2018).

El gráfico 1 muestra la evolución de la pobreza en el Estado de Hidalgo durante el período (2008-2018). En términos generales, la pobreza se redujo 11,4 puntos porcentuales en el período mencionado. Teniendo sobre todo un impacto considerable en el combate a la pobreza extrema, pasando de 15,3 puntos porcentuales en el año 2008 a 6,1 puntos 
porcentuales en 2018. No obstante, es necesario el desarrollo de una estrategia de combate a la pobreza sobre todo ante la actual situación pandémica. En este sentido, es preciso identificar la posición que ocupa Hidalgo en comparación con otras entidades. Por ejemplo, en el año 2008 se ubicaba en la séptima posición como uno de los treinta y dos Estados más pobres de México. En los años subsiguientes mejoró su ubicación hasta llegar en 2018 a la 12a. posición.

\section{Gráfico 1- Evolución de la pobreza en el Estado de Hidalgo} (2008-2018)
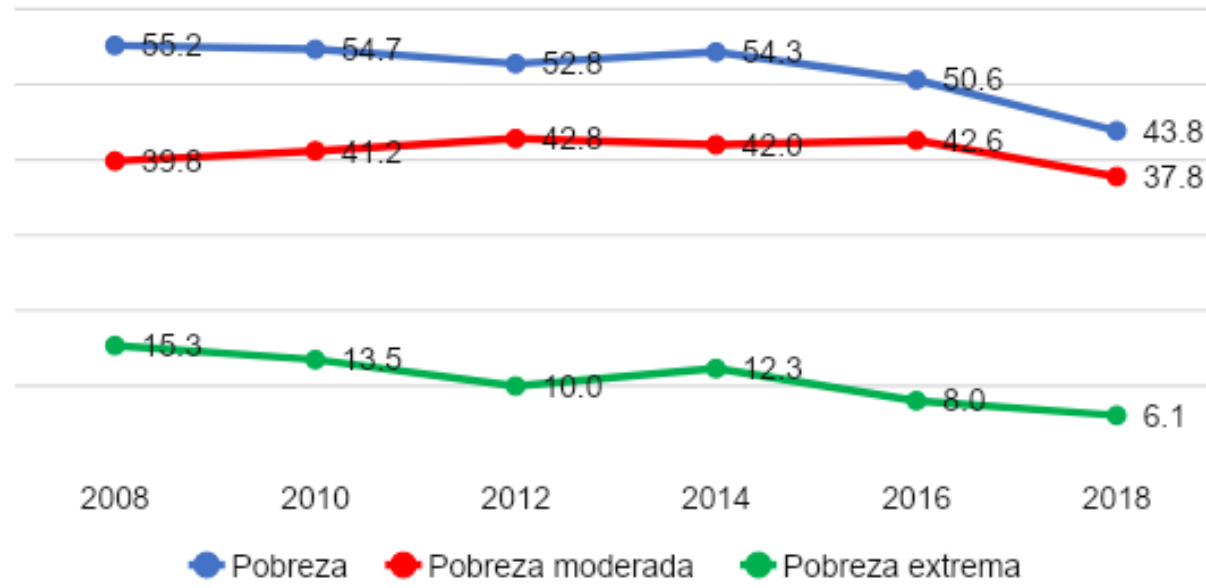

Fuente: elaboración propia a partir de anexo estadístico Hidalgo en CONEVAL (2017, n.p.).

Algunos indicadores de pobreza nos muestran de manera más clara el panorama de la entidad. Según datos del CONEVAL (2020, p.15), en 2018 solo un 13.5 \% de la población en Hidalgo no se encontraba en situación de pobreza, lo cual era equivalente a 403,500 personas. Ahora, si atendemos a la privación de carencias sociales ${ }^{4}$, para el mismo año un $82.7 \%$ de la población contaba con al menos una carencia social, siendo la principal la de acceso a la seguridad social, ya que para el año 2018 , un $70.8 \%$ de la población presentaba esta carencia. Y si bien, únicamente un $3.8 \%$ de la población era vulnerable por ingresos, aproximadamente un $47.7 \%$ de la población se ubicaba por debajo de la línea de pobreza por ingresos.

\footnotetext{
${ }^{4}$ EI CONEVAL (2020, p. 32) registra los siguientes indicadores de carencia social: a) Rezago educativo; b) Acceso a los servicios de salud; c) Acceso a la seguridad social; d) Calidad y espacios de la vivienda; e) Acceso a los servicios básicos de la vivienda; f) Acceso a la alimentación nutritiva y de calidad.
} 
Explicado desde el plano municipal, el CONEVAL ubicaba para el año 2015 a 64 de los 84 municipios con niveles de pobreza entre el rango del 40\% al 80\% del total de la población (2017). En tanto, únicamente catorce municipios estaban por debajo del 40\% y seis por encima del $80 \%$ (Tabla 1). En el caso de Tulancingo, se ubicaba en la posición cincuenta y nueve de los municipios más pobres con un 50.3\% de la población en esta condición.

Tabla 1- Municipios con un mayor número de personas en pobreza en el Estado de Hidalgo en 2015

\begin{tabular}{|c|c|c|c|}
\hline Municipio & Posición & Población total & $\begin{array}{c}\text { No. de personas en condición } \\
\text { de pobreza }\end{array}$ \\
\hline Pachuca de Soto & 1 & 292,931 & 88,306 \\
\hline $\begin{array}{c}\text { Tulancingo de } \\
\text { Bravo }\end{array}$ & 2 & 158,189 & 79,647 \\
\hline Huejutla de Reyes & 3 & 123,763 & 77,762 \\
\hline Ixmiquilpan & 4 & 94,569 & 52,584 \\
\hline Tizayuca & 5 & 121,366 & 41,798 \\
\hline
\end{tabular}

Fuente: elaboración propia a partir de anexo estadístico Hidalgo en CONEVAL (2017, n.p.).

\section{Progresa, oportunidades, Prospera y bienestar}

Los Programas de Transferencias Monetarias Condicionadas (PTC) se han convertido en una de las herramientas más utilizadas por los gobiernos Latinoamericanos para el combate a la pobreza. La aparición de los PTC se ubica a mediados de la década de 1990 (CEPAL/OIT, 2014; Cena; Chahbenderian, 2015). En el caso de México podemos remontarnos al decreto del 8 de agosto de 1997 a través del cual se crea la Coordinación Nacional del Programa de Educación, Salud y Alimentación (PROGRESA) (SEDESOL, 1999).

Posteriormente el Programa de Desarrollo Humano Oportunidades (PDHO) vendría a sustituir a PROGRESA. Este programa surgió mediante el decreto del 6 de marzo del 2002 (SEDESOL, 2002, n.p.). En su momento, el 
PDHO fue considerado como uno de los PTC de mayor importancia en Latinoamérica $^{5}$. Una de las características distintivas de este tipo de programas son las condicionalidades, las cuales deben ser cumplidas por las familias para mantener el apoyo económico proveniente del programa. En el caso del PDHO podemos mencionar las corresponsabilidades educativas y de salud (punto 3.6.2) (SEDESOL, 2013, n.p.).

Posteriormente, el 5 de septiembre de 2014 mediante decreto se crea la Coordinación Nacional de PROSPERA Programa de Inclusión Social (SEDESOL, 2014). Como podemos observar en el gráfico 2, la cobertura del programa PROSPERA alcanzó en 2018 a aproximadamente 6,5 millones de familias beneficiarias. Debemos mencionar que este programa contaba con los siguientes cuatro componentes: el educativo, de salud, alimentario y vinculación (SEDESOL, 2017, n.p.). La principal diferencia entre Oportunidades y Prospera, fue que este último buscaba una inclusión productiva, laboral, financiera y social de las familias beneficiarias.

Gráfico 2- Número de familias beneficiarias PROSPERA

(2014-2018)

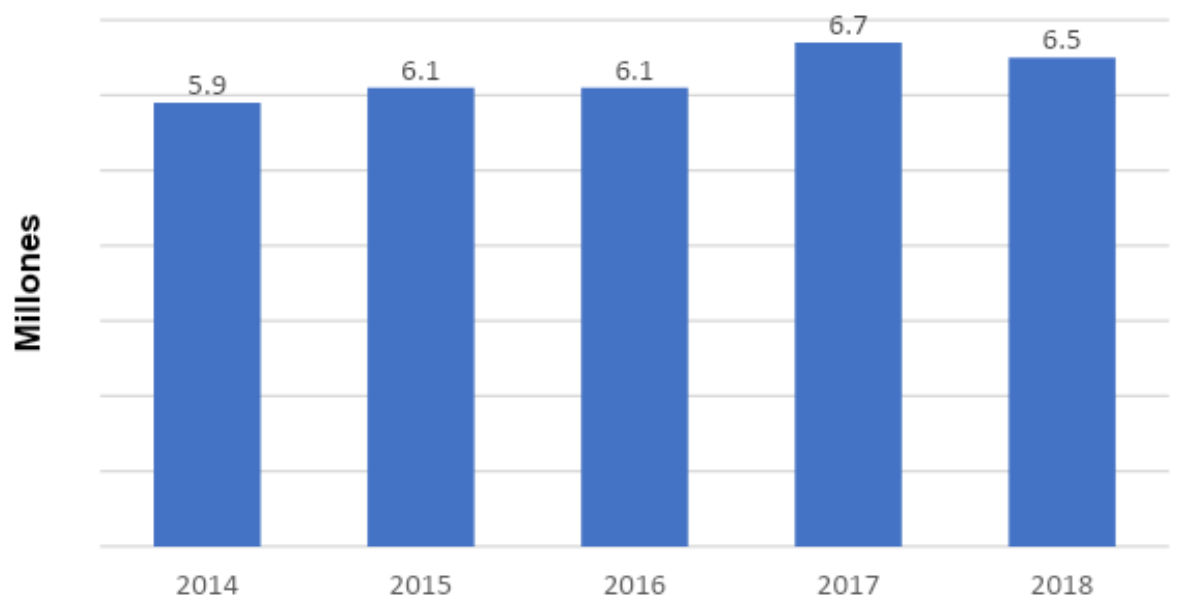

Fuente: elaboración del autor con datos de CEPAL (2021).

Con la entrada al gobierno de López Obrador en 2018, el programa Prospera desaparece en el año 2019. En su lugar se creó la Coordinación

\footnotetext{
${ }^{5}$ En 2003 el programa registró a 21,62 millones de personas beneficiarias, representativas de 4,24 millones de familias. Apenas un año después, se logró una cobertura de 25 millones de personas beneficiarias y un incremento a 5 millones de familias. En su último año en funcionamiento en 2014, alcanzaría a beneficiar a aproximadamente 6,1 millones de familias (CEPAL, 2021).
} 
Nacional de Becas para el Bienestar Benito Juárez mediante decreto del 31 de mayo del 2019 (SEP, 2019). La cual a través del Programa Becas Benito Juárez se conformó por cuatro modalidades: Beca Bienestar para las Familias de Educación Básica; Beca Universal para Estudiantes de Educación Media Superior Benito Juárez; Beca Jóvenes Escribiendo el Futuro de Educación Superior; y Becas Elisa Acuña. A diferencia de Prospera, no se exigen condicionalidades tales como: la asistencia escolar, registro en unidades de salud, asistencia periódica a citas médicas y participación en talleres comunitarios.

\section{$4 \quad$ Evolución de la pandemia y acciones de gobierno en méxico}

Según el informe "El impacto del Covid-19 en América Latina", se espera que la pandemia provoque la peor crisis sanitaria, económica, social y humanitaria de los últimos 100 años (NACIONES UNIDAS, 2020). Estimando que la tasa de pobreza aumentaría siete puntos porcentuales, hasta alcanzar el 37.2\%, mientras que la de extrema pobreza aumentaría 4,5 puntos porcentuales, del 11.0\% al 15.5\%, lo que representaría un incremento de 28 millones de personas (Ibid., p. 13). De igual forma se preveía que la fuerte caída de la actividad económica elevaría la tasa de desempleo del 8.1\% en 2019 al 13.5\% en 2020 (Id.).

Con respecto a México, el primer caso de Covid-19 fue confirmado el 28 de febrero del 2020. De esta manera se comenzaba la fase 1 de contingencia sanitaria ${ }^{6}$ (LÓPEZ OBRADOR, 2020a). En tanto, el 23 de marzo se iniciaba la "Jornada Nacional de Sana Distancia", un día después el gobierno federal declaraba la fase 2 de contingencia sanitaria. (LÓPEZ OBRADOR, 2020b). Posteriormente, el 30 de marzo, el Consejo de

\footnotetext{
${ }^{6}$ La Secretaría de Salud y el Instituto Nacional de Salud Pública (2020) consideran las siguientes tres fases de la epidemia por Covid-19: Fase 1 de importación del virus: las personas que se enfermaron viajaron al extranjero donde adquirieron la enfermedad y presentaron los síntomas a su regreso a México. Fase 2 de dispersión comunitaria: en esta fase existe transmisión de persona a persona independientemente de que hayan salido o no del país. Fase 3 de contagio epidémico: el número de casos aumenta a miles, los brotes son regionales y la dispersión de la enfermedad es a nivel nacional.
} 
Salubridad General anunciaba en México emergencia sanitaria. Como podemos observar la situación de deterioro en el país sucedió de manera acelerada, el 21 de abril de 2020 se iniciaba la fase 3 de contingencia sanitaria. (LÓPEZ OBRADOR, 2020c). A continuación, el 14 de mayo de 2020, el gobierno federal adoptó un semáforo epidemiológico para controlar las actividades en cada entidad (tabla 2) (SSA, 2020, n.p.).

Tabla 2- Semáforo por regiones

Actividades permitidas a partir del 1 de junio de 2020

\begin{tabular}{|c|c|c|}
\hline Región & Actividad & Descripción de actividades \\
\hline \multirow{3}{*}{ Rojo } & Escuelas & Suspendidas \\
\hline & Espacio público & Suspendidas \\
\hline & $\begin{array}{c}\text { Actividades económicas SOLO } \\
\text { ESENCIALES }\end{array}$ & Solo las actividades laborales consideradas esenciales \\
\hline \multirow{3}{*}{ Naranja } & Escuelas & Suspendidas \\
\hline & Espacio público & $\begin{array}{l}\text { Aforo reducido en las actividades del espacio público } \\
\text { en lugares abiertos. En lugares cerrados suspendidas. }\end{array}$ \\
\hline & $\begin{array}{l}\text { Actividades económicas } \\
\text { generales }\end{array}$ & $\begin{array}{l}\text { Actividades laborales consideradas esenciales y las } \\
\text { actividades no esenciales con una operación reducida. }\end{array}$ \\
\hline \multirow{3}{*}{ Amarillo } & Escuelas & Suspendidas \\
\hline & Espacio público & $\begin{array}{c}\text { Aforo permitido en las actividades del espacio público } \\
\text { en lugares abiertos y en lugares cerrados con } \\
\text { restricciones. }\end{array}$ \\
\hline & $\begin{array}{l}\text { Actividades económicas } \\
\text { generales }\end{array}$ & Todas las actividades laborales \\
\hline \multirow{3}{*}{ Verde } & Escuelas & Todas las actividades laborales \\
\hline & Espacio público & \multirow[b]{2}{*}{ Sin restricciones } \\
\hline & $\begin{array}{l}\text { Actividades económicas } \\
\text { generales }\end{array}$ & \\
\hline
\end{tabular}

Fuente: tabla extraída de la SSA (2020).

A partir del 1 de diciembre de 2020 el gobierno implementó la "Estrategia Nacional de Vacunación" por grupos poblacionales priorizados, la cual se tiene previsto finalizar en marzo del 2022 (ver gráfico 3). No obstante, podemos mencionar que se ha anticipado la vacunación de otros grupos poblacionales. Por ejemplo, del personal del sector educativo alrededor de 3, 03 millones de personas durante el período del 20 de abril al 28 de mayo del 2021, esto para buscar el regreso a clases presenciales (EL 
UNIVERSAL, 2021). De igual forma, se vacunará a la brevedad a las mujeres embarazadas con gestación posterior a la novena semana (LÓPEZ OBRADOR, 2021). Hasta el día 11 de mayo del 2021 se habían aplicado en México 21, 358,971 dosis (Id.).

Gráfico 3. Etapas de la estrategia de vacunación por grupos poblacionales priorizados

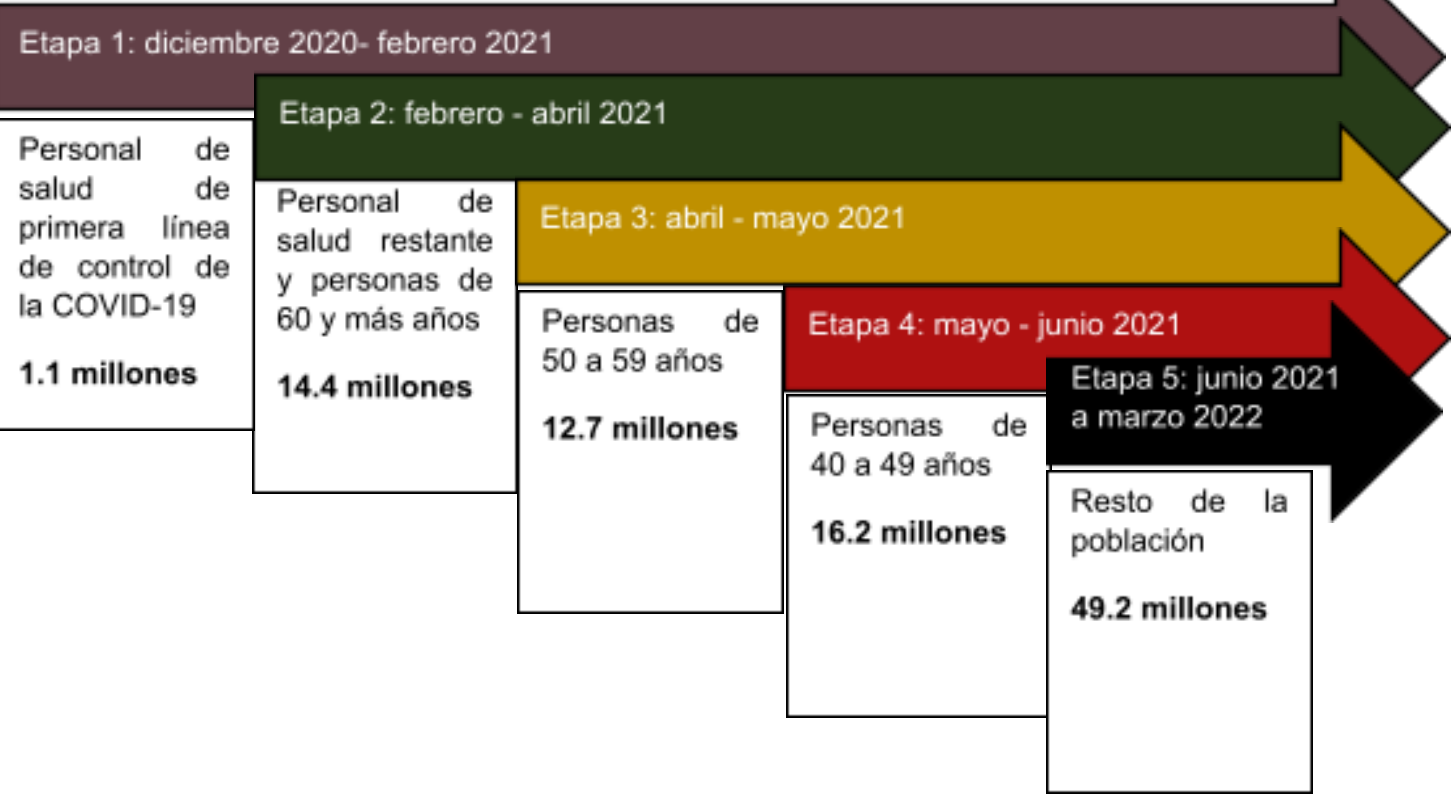

Fuente: imagen extraída de Cortés; Gómez; Alba (2021, p. 21)

\section{$5 \quad$ El caso del municipio de Tulancingo de Bravo}

5.1 La desaparición de Prospera y la transición al Programa de Becas Benito Juárez

Las mujeres entrevistadas fueron beneficiarias de algún PTC 7 al menos desde el año 2013, al momento de la desaparición de Prospera en 2019 habían recibido una transferencia monetaria condicionada ${ }^{8}$ por un mínimo de seis años. Al finalizar el programa Prospera, las mujeres entrevistadas

\footnotetext{
7 Según Parker (2019), PROSPERA ha mostrado resultados positivos en los indicadores de educación, salud y nutrición. Con respecto a los niños menciona que se ha logrado aumento en la escolaridad y la salud. Por otro lado, en la siguiente generación señala que se muestran efectos positivos en los resultados laborales de los jóvenes, pero poca movilidad ocupacional.

8 Parker (2019) presume que otorgar las transferencias monetarias a la madre o la jefa del hogar mejora su situación frente a los hombres en el hogar. En tanto, Ochman (2016) sostiene que los PTC promueven cierta independencia económica a la mujer, pero reproducen estereotipos y roles tradicionales, generando impacto negativo en su bienestar.
} 
manifestaron que pasaron a formar parte del Programa de Becas Benito Juárez en alguna de sus modalidades. De igual manera mencionaron que notaron principalmente dos cambios durante la transición entre ambos programas: la eliminación de las condicionalidades para recibir el apoyo y la periodicidad de entrega del recurso económico.

En cuanto a las condicionalidades ${ }^{9}$, señalaron que para recibir el apoyo de Prospera, los integrantes de la familia debían acudir a consultas médicas cada seis meses, en el caso de los hijos asistir a la escuela y ellas tomar talleres con compañeras del programa. No debemos olvidar que en 2016 registramos casos de sobrecarga de actividades ${ }^{10}$ debido a las condicionalidades que se exigían en Prospera. En la actualidad parece ser que la eliminación de las condicionalidades está provocando ciertos efectos tanto positivos como negativos. Con respecto a los primeros, las mujeres comentan que pueden trabajar tranquilamente sin tener que pedir permiso para asistir a juntas o citas médicas. Sin embargo, también han surgido desventajas, por ejemplo, los miembros de las familias ya no asisten periódicamente a revisiones médicas, y algunas de las entrevistadas han manifestado que extrañan los talleres y han dejado de tener contacto con las otras mujeres.

Con respecto a las condicionalidades mencionan lo siguiente:

Erika: "con Prospera, teníamos las consultas, peso y talla, íbamos a talleres con compañeras, con las enfermeras, nos mandaban a traer al auditorio para darnos un tema las vocales y desde que pasó eso (desaparición de Prospera), ya no supimos nada..."

Cristina: "nos entregan un apoyo para los hijos que estudian (Programa de Becas Benito Juárez), pero antes nos daban un apoyo igual por parte de Prospera, pero para salud, alimentación, y estudios, y ahora esta abarca solo estudios y es por familia, no es por hijos".

\footnotetext{
${ }^{9}$ El programa Prospera contaba con condicionalidades educativas y de salud. De acuerdo al punto 3.8.3, su cumplimiento era requisito indispensable para recibir los apoyos monetarios (SEDESOL, 2015, n.p.).

10 Si bien la mujer es la receptora del beneficio, es también la principal encargada del cumplimiento de las condicionalidades (MEZA et al., 2002; GAMMAGE, 2010). Por lo que, combinar una "doble jornada" o "doble presencia", es decir, compaginar un trabajo remunerado y el cuidado del hogar, con la observancia de las condicionalidades parece generar una sobrecarga de actividades (CECCHINI; MADARIAGA, 2011).
} 
Cristina: "recibía un apoyo para mi hija de alimentación, recibía papillas, iba al centro de salud, ahorita no tengo ninguno de esos beneficios"

Laura: "a mí me gustaba antes (con Prospera), nos daban más enseñanzas, nos enseñaban a cuidarnos a nosotras mismas, nos daban talleres de violencia, si pasaba algo de violencia, era un factor de riesgo, pero pues ahora ya no hay esos talleres, a mí en lo personal me gustaba ir a esos talleres..."

La periodicidad en la entrega de los apoyos del nuevo gobierno ha representado uno de los puntos más repetidos a lo largo de las entrevistas, derivado de la falta de puntualidad en la entrega de los apoyos, ya que con Prospera las transferencias monetarias ${ }^{11}$ se realizaban puntualmente. Por ejemplo, en el caso de Luisa, señala que desde octubre del 2020 no ha recibido nada, si tomamos en cuenta que se le entrevistó en marzo de 2021, eso quiere decir que lleva aproximadamente cinco meses sin recibir ningún beneficio, la situación parece agravarse si agregamos que nos encontramos en pandemia.

Las entrevistadas comparan la periodicidad de entrega del apoyo económico de ambos programas de la siguiente forma:

Cristina: "pues ha empeorado (su situación), porque le comento que al menos sabíamos que llegaba un apoyo bimestral (refiriéndose a Prospera) y era exacto, ahorita, por ejemplo, a nosotras aquí en la localidad, nos traen que mañana, que pasado..."

Monserrat: "antes era cada dos meses (refiriéndose a Prospera), ahorita ya no se sabe, nos dicen para una fecha y luego no llega y llega después".

Luisa: "en Prospera llegaba puntual exactamente cada dos meses estaba nuestro apoyo, ahora yo en Bienestar la última vez que recibí mi apoyo fue en octubre".

5.2 El impacto del Covid-19 en la educación, el empleo y la salud

Si bien el gobierno federal ha diseñado una serie de medidas preventivas contra el Covid-19, los Estados han implementado medidas complementarias. Entre algunas de las acciones del gobierno de Hidalgo

\footnotetext{
"De acuerdo al punto 3.7.1, los apoyos monetarios directos y las becas educativas se entregaban bimestralmente (SEDESOL, 2017, n.p.).
} 
tenemos la instalación del "Hospital de Respuesta Inmediata Covid-19" mejor conocido como "Hospital inflable" que se llevó a cabo el 14 de marzo del 2020 en Pachuca de Soto (ANGELES, 2020). De igual forma, podemos mencionar el "Operativo Escudo" implementado a partir del 16 de marzo del mismo año (REYES, 2020).

Con respecto a apoyos económicos ${ }^{12}$ han sido escasos en todos los niveles de gobierno. En general las mujeres entrevistadas han mencionado desconocer y no haber recibido ningún tipo de beneficio derivado de la situación provocada por la pandemia. Únicamente una de las mujeres entrevistadas señala haberse enterado del "apoyo a deudos de fallecidos por Covid-19"13, esto debido al deceso de su madre. Por otra parte, Laura menciona haber escuchado que en caso de fallecimiento de algún familiar, el gobierno otorgaba un apoyo económico. No obstante, desconoce si sea federal, estatal o municipal, además de que ella lo considera un rumor.

Lo cual expresa de la siguiente manera:

Cristina: "Lo de los gastos funerarios de Covid sí, sí se dio, y yo creo que es el único apoyo que se ha recibido por Covid, pero lamentablemente es por un fallecimiento".

Uno de los principales efectos del Covid-19 lo podemos observar en el aspecto educativo. Las clases dejaron de ser presenciales y pasaron a ser a distancia en prácticamente todos los niveles educativos. El gobierno mexicano suspendió las clases $^{14}$ a partir del 23 de marzo y hasta el 17 de abril del 2020 en las escuelas dependientes de la Secretaría de Educación Pública (SEP, 2020, n.p.). El plazo ha continuado extendiéndose en la mayoría de los Estados del país. No obstante, se espera un pronto regreso a

\footnotetext{
${ }^{12}$ La alcaldía de Pachuca de Soto lanzó una convocatoria mediante la cual brindaría apoyos a 500 negocios con un monto de 4 mil pesos (US\$ 178.33 al 11 de agosto del 2020) a fondo perdido para la reactivación económica (Garmez, 2020). De igual forma, para el mes de marzo del 2021 el cabildo de Pachuca otorgaría apoyos de 3 mil a 15 mil pesos (US\$ 145.20 a US\$ 726.04 al 1 de marzo del 2021) a comerciantes y locatarios. Además del empleo de 1,5 millones de pesos (US\$ 72,604.06 al 1 de marzo del 2021) para adquirir una ambulancia equipada (Jiménez, 2021). Con respecto a Tulancingo de Bravo no parece existir registro de algún apoyo económico.

${ }^{13}$ En conferencia matutina del 24 de noviembre del 2020, el director general del Instituto Mexicano del Seguro Social, Mtro. Zoé Robledo anunció un apoyo del gobierno federal para deudos de fallecidos por Covid-19 por un monto de 11, 460 pesos (US\$ 572.14 al 24 de noviembre del 2020) (LÓPEZ OBRADOR, 2020d).

${ }_{14}$ Según estimaciones de UNICEF (2020), alrededor de 154 millones de niños, niñas y adolescentes, es decir, un 95 por ciento de los matriculados en la región de América Latina y el Caribe, permanecían fuera de las escuelas a causa del COVID-19.
} 
clases presenciales ya que como se mencionó el gobierno ha puesto en marcha la vacunación del personal educativo.

Según datos de INEGI, de la totalidad de los hogares del país, 44.3\% dispone de computadora, y 92.5\% cuenta con al menos un televisor (2021a, p. 1). En tanto se estima que 86,5 millones de personas cuentan con un teléfono celular, lo que es equivalente a un $75.1 \%$ de la población del país (Ibid., p. 2). Las mujeres que hemos entrevistado cuentan con al menos dos hijos menores de dieciocho años de edad que se encuentran estudiando, solo en el caso de Monserrat tiene tres hijos. Tal y como señalan, ninguna de ellas contaba con equipo de cómputo ni con servicio de internet previo a la transición a clases en línea, esto puede atribuirse a su condición de pobreza. Algunas de ellas optaron en un primer momento por poner recargas de saldo a sus celulares o comprar algún aparato móvil para que sus hijos pudieran tomar clases en línea.

Una de las entrevistadas menciona lo siguiente:

Erika: "ella tenía un celular (su hija) pero que le regalamos hace años, y pues ese celular no podíamos descargarle el Classroom, el Meet todo eso que están usando, mi esposo tuvo que hacer el esfuerzo de sacarle un celular más grande para que pudiera descargarle todo. Y ahora teníamos el problema de que las recargas no eran suficientes, porque no abastecían toda su semana, entonces tuvimos que contratar internet".

Debido al costo elevado de poner recargas de saldo a los celulares, las cuales iban desde 50 hasta 250 pesos por semana (US\$2.51 a US\$12.54 al 31 de mayo del 2021), algunas de ellas decidieron contratar servicios de internet. Por ejemplo, en el caso de Erika menciona que por semana hacía una recarga de saldo de 50 pesos. No obstante, refiere que no era suficiente para abastecer toda la semana, y que a veces sus hijos no podían entregar las tareas. O en el caso de Laura, ella cita que las videollamadas se cortaban por falta de saldo. Por otro lado, Monserrat gastaba 50 pesos por su hija, otros 50 pesos por su hijo y ella 100 pesos semanales (US\$ 5.01 al 31 de mayo del 2021). Esto las orilló a casi todas a contratar servicios de internet con un costo mínimo de 400 hasta 550 pesos mensuales (US\$ 
20.06 a US\$ 27.58 al 31 de mayo del 2021). Solo en el caso de Ana su situación económica le ha impedido contratar el servicio. Tampoco ninguna de ellas ha comprado un equipo de cómputo.

Con respecto al aprendizaje ${ }^{15}$ pudimos percatarnos que se aducen ciertas dificultades. Por ejemplo, Erika refiere problemas en materias como matemáticas, o en los casos de Ana y Laura mencionan que ellas se desesperan al no entender algunas tareas y no poder ayudar a sus hijos. Encontramos también referencias al estrés ${ }^{16}$ provocado por sobrecarga de tareas. Lo que las lleva a preferir que estos regresen a clases de manera presencial. A pesar de esto, encontramos la mención de ciertas ventajas de clases en línea tales como: menos gasto en transporte, ahorro en comida, mayor convivencia familiar y seguridad de los hijos al estar en casa.

Algunas mujeres señalan lo siguiente:

Laura: "ya ahora con el paso de los días y las tareas ya mejor que estén en las clases (presenciales)".

Monserrat: "luego si nos ponían muchas cosas, yo digo las maestras no lo hacen en las escuelas y quieren que los papás nos pongamos ahí sabiendo que nosotros tenemos que trabajar".

En el caso de México, apenas en junio del 2020 la tasa de desempleo subió a 5.5\%. De igual forma en el segundo trimestre del mismo año, se reportó una caída del PIB de $-17.3 \%$, esto debido a la emergencia sanitaria (FEIX, 2020). Recientemente el INEGI (2021b, p.1) informó sobre los resultados de la Encuesta Nacional de Ocupación y Empleo (ENOE) de marzo de 2021, señalando que se presentó una disminución de 1,5 millones de personas de la Población Económicamente Activa (PEA) en comparación con marzo del 2020.

\footnotetext{
${ }^{15}$ CEPAL/UNESCO (2020), señalan que todavía no es posible determinar con certeza el impacto que tendrá la crisis en la implementación curricular en los distintos grados de la enseñanza primaria y secundaria. Sin embargo, se prevé una profundización de las diferencias en logros de aprendizaje.

${ }^{16}$ Con respecto al impacto psicológico y socioemocional en la comunidad educativa, CEPAL/UNESCO (2020), mencionan que el confinamiento significa para gran parte de la población vivir en hacinamiento, el cual por un período prolongado puede traer graves implicaciones para la salud mental, además de la exposición a situaciones de violencia para los niños, niñas y adolescentes.
} 
Con respecto a las mujeres entrevistadas algunas nos dejan ver que la pandemia ha afectado su situación laboral ${ }^{17}$. Por ejemplo, en el caso de Monserrat en 2016 siendo beneficiaria de Prospera no trabajaba, pero al salir de la casa de su suegra se vio en la necesidad de buscar un empleo remunerado. Menciona que en un inicio buscó vender ropa por medio de la plataforma Facebook teniendo muy buenos resultados. No obstante, su situación cambió con la llegada de la pandemia de la siguiente manera:

Monserrat: "Yo igual vendo ropa por Facebook, igual me afectó (la pandemia), todavía me afecta, hay muchos lugares en donde yo tengo que asistir a las plazas para surtirme y comprar ropa, y pues ahorita con esto hay muchas plazas que las cerraron y hay veces que no encuentro, por eso fue que me tuve que meter a trabajar en casa (empleada doméstica)".

Tal como pudimos constatar en la entrevista, también se vio perjudicada en el trabajo de empleada doméstica. En un inicio ayudaba a tres señoras, pero ante el aumento de casos de infección por la pandemia, una de sus empleadoras enfermó y otra debido al temor de contagio prescindió de sus servicios. Además, su esposo que trabajaba en un taller de hojalatería y pintura, en cierto momento lo mandaron a descansar a casa sin ningún tipo de sueldo por mes y medio. De tal forma que el dinero que ella obtenía era el único sustento del hogar.

En los casos de Laura, Cristina y Erika ninguna de ellas se encuentra laborando en la actualidad, son amas de casa. No obstante, han resentido los efectos de la pandemia, por ejemplo, el esposo de Laura es albañil, antes trabajaba semana completa y ahora únicamente tres o cuatro días por semana. En cambio, a la pareja de Cristina que trabaja en serigrafía, le han dejado de enviar trabajo y por lo tanto no recibe ingresos, en ocasiones labora dos o tres días por semana. Incluso cuando cerraron las fábricas le retuvieron el pago por trabajo ya realizado, por lo que para poder

\footnotetext{
${ }^{17}$ Según informe publicado por OIT (FEIX, 2020), entre marzo y abril del 2020 la tasa de informalidad se redujo 8 puntos porcentuales, del 55,7\% al 47,7\%. Lo que se tradujo en una pérdida de 10,4 millones de puestos informales. Sin embargo, durante los meses de junio, julio y agosto se recuperó hasta alcanzar un $55,1 \%$ de los ocupados. Con respecto al empleo formal en el período entre marzo y julio se perdieron 1,1 millones de empleos formales.
} 
sostenerse económicamente tuvieron que vender un terreno. El caso de Erika es particular, su esposo arregla radiadores con un familiar, de tal manera que no le han descontado ni dejado de pagar sin importar la disminución de trabajo. De igual forma nos menciona que en un inicio no cerraban a las horas especificadas por el gobierno, pero ante las advertencias de clausura tuvieron que acatar las indicaciones. Tanto en el caso de Luisa como el de Ana mencionan que no han tenido reducciones de sueldo ni las han mandado a casa a descansar.

Según datos encontrados en la página oficial "COVID-19-Gobierno del Estado de Hidalgo", al día 16 de mayo del 2021 se habían registrado en Tulancingo de Bravo 2, 751 casos positivos acumulados y 430 defunciones resultado del Covid-19 (HIDALGO, 2021). Las mujeres entrevistadas mencionaron que aplican las siguientes medidas preventivas: el uso de cubrebocas, tener gel antibacterial, salir solo en caso necesario ya sea por trabajo o compras de despensa, no visitar a nadie, limpiar el interior del hogar constantemente, desinfectar productos que traen de la calle, mudar y lavar ropa cada vez que regresan del exterior.

Entre las personas entrevistadas tenemos casos de infección y fallecimiento de familiares. Los hogares de Ana, Cristina, Luisa y Monserrat se han visto inmersos en alguna de las situaciones anteriores. En el caso de Cristina, nos encontramos con el fallecimiento de tres de sus familiares entre julio y agosto del 2020. Debido a la condición de pobreza, el poder solventar el aspecto económico es complicado. La entrevistada señala que para afrontar los gastos médicos y funerarios tuvieron que hacer uso de los ahorros que tenían y vender un terreno, además menciona que gastaron aproximadamente entre 60 a 70 mil pesos (US\$2,729.75 a 3,184.71 al 22 de agosto del 2020)

Ella lo narra de la siguiente forma:

Cristina: "al principio la verdad no creíamos (en el Covid-19), ya después, aquí en casa sí tocó pandemia...después de que nos enfermamos, sanitizamos..." 
Cristina: "fallece su papá (refiriéndose a su esposo), van al doctor (su esposo y suegro), fueron al doctor y les dijeron que era su azúcar, y no nos cuidamos, teníamos el cubrebocas todos, pero pues él comenzaba a trasudar, todo eso recibimos, la enfermedad..."

Cristina: "el día que se va a sepultar (a su suegro) y como dijeron que no era Covid, lo fueron a sepultar, hicieron aquí el rezo (en casa de ella) y después se lo llevaron a sepultar. Entonces, mi mamá ya se empezaba a sentir mal... a los cuatro días mi esposo cae en cama... y a los dos días estaba con oxígeno (su esposo) ..."

Cristina: "mi suegro era adulto mayor, tenía 72 años, mi suegra igual falleció tenía 68 años...mi mamá fallece el 22 de agosto tenía 61 años..."

Otro caso similar al anterior es el de Ana, ella refiere que su esposo es taxista y para prevenir algún contagio colocaba en su unidad un hule para separar al conductor del pasajero, además utilizaba cubrebocas y gel antibacterial. No obstante, se infectó en enero del 2021, y posteriormente todos los integrantes de su hogar también se contagiaron. Por otro lado, es preciso mencionar que su esposo está enfermo de insuficiencia renal y tiene que realizarse dos sesiones de hemodiálisis cada semana con un valor de 1, 200 pesos cada una (US\$58.50 al 31 de enero del 2021). Debido a que se infectó de Covid-19 lo remitieron a otro hospital para seguir con sus sesiones, las cuales tuvieron un costo de 3,400 pesos cada una (US\$ 165.77 al 31 de enero del 2021), en total se realizó ocho sesiones antes de recuperarse del Covid-19. Ana menciona que para afrontar los gastos tuvieron que empeñar casi todo, incluso dejaron su casa en garantía. Afortunadamente todos los miembros de su hogar lograron superar la enfermedad.

A continuación, se observa un extracto de la entrevista de Ana:

Ana: "mi esposo hace un año enfermó de insuficiencia renal...él va a sus hemodiálisis, va dos veces por semana precisamente por la economía porque cada una vale 1,200 pesos, no contamos con seguro ni con nada...ya cuando estaba en su sesión de hemodiálisis él 
se sintió mal, lo checan los doctores y tenía la presión y la azúcar alta y tenía temperatura de casi $40^{\circ} \mathrm{C} . . . "$

Ana: "el doctor le manda a hacer la prueba de Covid (costo de 3, 400 pesos) y le sale positiva...a los dos o tres días comencé a estar mal... mis niños también empezaron y enseguida mi suegra... mi hermana hasta usó oxígeno..."

Ana: "yo pensé que mi esposo por su enfermedad no la iba a librar (sobrevivir)..."

En el caso de Luisa y Monserrat no se presentaron complicaciones de salud por el contagio de Covid-19, pero al igual que los dos casos anteriores existieron dificultades económicas para solventar los gastos. Únicamente Laura y Erika mencionan que no ha habido confirmación de contagio de ninguno de sus familiares. Sin embargo, todas ellas argumentan que tienen pensado vacunarse en cuanto se encuentre disponible la vacuna.

\section{Reflexiones finales}

Con respecto al año 2016, la condición de las mujeres entrevistadas no parece haber mejorado significativamente al punto de abandonar la pobreza, ya que siguen siendo beneficiarias de programas de gobierno. Al finalizar PROSPERA, estas mujeres no se vieron desprotegidas, ya que pasaron a formar parte del Programa Becas para el Bienestar Benito Juárez. Lo que les permitió seguir contando con un apoyo económico del gobierno y en un inicio les brindó cierta tranquilidad. No obstante, el paso de un programa a otro trajo consigo principalmente dos cambios: la eliminación de condicionalidades y un desfase en la entrega de los apoyos. Si bien se han tenido ventajas y desventajas con la desaparición de las corresponsabilidades, estas han quedado en un segundo plano, ya que para las mujeres entrevistadas el retraso en la entrega del apoyo económico se convierte en prioritario, esto debido a su situación de pobreza y la emergencia sanitaria generada por el Covid-19. Por lo tanto, es necesario que el gobierno emprenda acciones para cumplir con la entrega de las transferencias monetarias. 
En educación, la transición de clases presenciales al formato a distancia ha provocado una serie de cambios en diferentes aspectos. Con respecto al económico, algunas familias se han visto en la necesidad de realizar recargas de saldo a móviles, otras contratar el servicio de internet o comprar algún aparato móvil a plazos. Esto sin existir ningún apoyo de gobierno en atención a lo anterior. Si tomamos en cuenta que estas familias son de escasos recursos, el gasto erogado genera un impacto considerable. En tanto, en el aprendizaje nos encontramos con estrés en los menores, además las mujeres entrevistadas consideran que sus hijos no están aprendiendo adecuadamente y algunas sugieren el regreso a clases presenciales.

De igual forma otro de los impactos del Covid-19 se puede ver reflejado en el sector laboral. Como se ha visto, en el caso de México ha aumentado la tasa de desempleo. Por otra parte, en algunos de los casos estudiados, se observa una reducción en la demanda de trabajo, que conllevó a una disminución de los ingresos familiares. En cuanto al aspecto de salud el Covid-19 generó graves repercusiones sobre todo para las personas en condiciones de pobreza. Como hemos revisado al ser un gasto no previsto algunas de ellas se vieron en la necesidad de vender el poco patrimonio que tenían, pedir prestado y empeñar objetos.

Con respecto a los distintos niveles de gobierno existen escasos programas de apoyo a la economía familiar derivados de la situación pandémica. Aunado a la aparente desinformación entre la población acerca de estos apoyos. Si bien el plan de vacunación en México está avanzando conforme a lo planeado, sin duda el principal reto del gobierno será enfrentar una reactivación económica que tenga como punto de partida a los grupos más vulnerables. 
ANGELES, Verónica. Instalan hospital inflable en Pachuca para atender casos de coronavirus. El Sol de México. Publicado en: 14 mar. 2020. Disponible en: https://www.elsoldemexico.com.mx/republica/sociedad/instalan-hospital-inf lable-en-pachuca-hialgo-para-atender-casos-de-coronavirus-4971439.html Acceso en: 17 abr. 2021.

BADILLO FLORES, Pastor. Cash transfer programmes as a means to women's empowerment? The Oportunidades Cases in Tulancingo, Mexico (2002-2014). In: CRUZ, Gibrán (ed). Welfare and Social Protection in Contemporary Latin America. New York: Routledge, 2019. p.198-214.

CECCHINI, Simone; MADARIAGA Aldo. Programas de Transferencias Condicionadas: Balance de la experiencia reciente en América Latina y el Caribe. Santiago: Naciones Unidas, CEPAL y ASDI, 2011. Disponible en: https://repositorio.cepal.org/bitstream/handle/11362/27854/1/S2011032_es.pdf . Accedido en: 15 feb. 2021.

CENA, Rebeca; CHAHBENDERIAN, Florencia. El abordaje estatal de la pobreza en Programas de Transferencias Monetarias Condicionadas. Revista Latinoamericana de Ciencias Sociales, Niñez y Juventud, v.13, n1, p. 123-136. 2015. DOI:10.11600/1692715x.1316260914.

CEPAL. Programas de Transferencias Condicionadas. Base de datos de Programas de protección social no contributiva en América Latina y el Caribe. [s.i.]. Disponible en: https://dds.cepal.org/bpsnc/ptc. Accedido en: 16 mar. 2021.

CEPAL/OIT. Coyuntura Laboral en América Latina y el Caribe: los programas de transferencias condicionadas y el mercado laboral. n.20. Santiago: Naciones Unidas, 2014. Disponible en: https://www.cepal.org/es/publicaciones/36896-coyuntura-laboral-america-I atina-caribe-programas-transferencias-condicionadas. Accedido en: 18 mar. 2021.

CEPAL/UNESCO. Informe COVID-19: La educación en tiempos de pandemia. 20 ago. 2020. Disponible en: https://repositorio.cepal.org/bitstream/handle/11362/45904/1/S2000510_es.p df. Accedido en: 5 sept. 2021.

CONEVAL. Medición de la pobreza, Hidalgo, 2010-2015. Anexo estadístico Hidalgo. Ciudad de México: CONEVAL, 2017. Disponible en: https://www.coneval.org.mx/Medicion/Paginas/AE_pobreza_municipal.aspx. Accedido en: 19 mar. 2021.

CONEVAL. Informe de pobreza y evaluación 2020: Hidalgo. Ciudad de México: CONEVAL, 2020. Disponible en: https://www.coneval.org.mx/coordinacion/entidades/Documents/Informes_ 
de_pobreza_y_evaluacion_2020_Documentos/Informe_Hidalgo_2020.pdf Accedido en: 19 mar. 2021.

CORTÉS, Ricardo; GÓMEZ, Raúl; ALBA, Xiomara. Política Nacional de Vacunación contra el virus SARS-CoV-2, para la prevención de la Covid-19 en México. $1^{a}$.ed., versión 4.0. Ciudad de México: Gobierno de México. Publicado en: 11 ene. 2021. Disponible en: https://coronavirus.gob.mx/wp-content/uploads/2021/01/PolVx_COVID_-11En e2021.pdf. Accedido en: 18 out.2021.

EL UNIVERSAL. La Mañanera de AMLO, viernes 16 de abril del 2021. YouTube. Publicado en: 16 abr. $2021 . \quad$ Disponible en: https://youtu.be/gUrPbXzoxho. Accedido en: 25 abr. 2021.

FEIX, Noémie. México y la crisis de la COVID-19 en el mundo del trabajo: respuestas y desafíos. [S.I.]: OIT, 2020. Disponible en: https://www.ilo.org/mexico/publicaciones/WCMS_757364/lang--es/index.ht m. Accedido en: 1 sept. 2021.

GAMMAGE, Sarah. Time Pressed and Time Poor: Unpaid Household Work in Guatemala. Feminist Economics, v.16, n.3, p.79-112, 2010. Disponible en: DOI.org/10.1080/13545701.2010.498571. Accedido en: 18 out. 2021.

GARMEZ, Adela. Así podrán acceder a apoyo de 4 mil pesos negocios y mercados de Pachuca Criterio Hidalgo. Publicado en: 11 ago. 2020. Disponible en: https://criteriohidalgo.com/destacado/asi-podran-acceder-apoyo-4-mil-pes os-negocios-mercados-pachuca. Accedido en: 18 abr. 2021.

HIDALGO. COVID-19-Gobierno del Estado de Hidalgo, 2021. Página inicial. Disponible en: https://coronavirus.hidalgo.gob.mx/ Accedido en: 21 mar. 2021.

HIGGINS, Sean. The impact of Bolsa Familia on poverty: does Brazil's Conditional Cash Transfer Program have rural bias? Journal of Politics \& Society, verano, 2012, pp.88-125. Disponible en: DOIrg/10.7916/D8B27S8K. Accedido en: 25 mar. 2021.

JIMÉNEZ, Alan. Promete alcaldía de Pachuca apoyo económico y ambulancia para comerciantes. Quadratín Hidalgo, 1 mar. 2021. Disponible en:

https://hidalgo.quadratin.com.mx/municipios/promete-al-alcaldia-de-pach uca-apoyo-economico-y-ambulancia-para-comerciantes/. Accedido en: 25 mar. 2021.

INEGI. Cuéntame de México. Mapoteca. Mapa de Hidalgo. División municipal, 2018.2 Disponible en: http://cuentame.inegi.org.mx/mapas/pdf/entidades/div_municipal/hgo.pdf. Accedido en: 26 mar. 2021.

INEGI. Comunicado de prensa núm. 103/20. INEGI, IFT, Secretaría de Comunicaciones y Transportes. Publicado en: 17 feb. 2020. Disponible en: 
https://www.inegi.org.mx/contenidos/saladeprensa/boletines/2020/OtrTemE con/ENDUTIH_2019.pdf Accedido en: 6 sept. 2021.

INEGI. Panorama sociodemográfico de Hidalgo: Censo de Población y Vivienda 2020. Aguascalientes: CPV, Instituto Nacional de Estadística y Geografía, INEGI, 2021a. Disponible en: https://inegi.org.mx/contenidos/productos/prod_serv/contenidos/espanol/bv inegi/productos/nueva_estruc/702825197865.pdf Accedido en: 27 mar. 2021.

INEGI. Comunicado de prensa núm. 216/21. INEGI. Publicado en: 22 abr. 2021b. Disponible en: https://www.inegi.org.mx/contenidos/saladeprensa/boletines/2021/iooe/iooe 2021_04.pdf. Accedido en: 6 sept. 2021.

LÓPEZ OBRADOR, Andrés Manuel. México está preparado ante el coronavirus. Conferencia presidente AMLO. YouTube, 28 feb. 2020a. Disponible en: https://youtu.be/MmkzX7372RO Accedido en: 16 may. 2021.

LÓPEZ OBRADOR, Andrés Manuel. Anuncio de la fase 2 de Covid-19. Conferencia presidente AMLO. YouTube, 24 mar. 2020b. Disponible en: https://youtu.be/ApckBHmoOsc Accedido en: 16 may. 2021.

LÓPEZ OBRADOR, Andrés Manuel. Inicio de la fase 3 de la epidemia de Covid-19. Conferencia presidente AMLO. YouTube, 21 abr. 2020c. Disponible en: https://youtu.be/UzMDQkSqkWg. Accedido en: 16 may. 2021.

LÓPEZ OBRADOR, Andrés Manuel. Apoyo para gastos funerarios por COVID-19. Conferencia presidente AMLO. YouTube, 24 nov. 2020d. Disponible en: https://youtu.be/rRXYSNeltcs. Accedido en: 16 may. 2021.

LÓPEZ OBRADOR, Andrés Manuel. Inicia registro de embarazadas para vacunación contra COVID-19. Conferencia presidente AMLO. YouTube, 11 may. 2021. Disponible en: https://youtu.be/6oDtthq3xFM. Accedido en: 16 may. 2021.

MEZA, Alejandro., et al. "Progresa" y el empoderamiento de las mujeres: estudio de caso en Vista Hermosa, Chiapas. Papeles de Población, v.8, n.31, pp. 67-93, ene.-mar. 2002. Disponible en: http://www.scielo.org.mx/scielo.php?script=sci_arttext\&pid=S1405-74252002 000100004. Accedido en: 5 sept. 2021.

NACIONES UNIDAS. Informe: El impacto del Covid-19 en América Latina. 2020. Lima: UN, 2020. Disponible en: https://peru.un.org/es/52494-el-impacto-del-covid-19-en-america-latina. Accedido en: 30 ago. 2021.

OCHMAN, Marta B. Políticas Sociales y empoderamiento de las mujeres. Una promesa incumplida. Estudios Políticos, v.48, pp.32-51, 2016. Disponible en: DOI.org/10.17533/udea.espo.n48a03.

PARKER, Susan W. La evaluación de Progresa-Oportunidades-Prospera: logros después de veinte años y retos para el futuro. In: HERNÁNDEZ LICONA, Gonzalo; et al. (coords.) . El Progresa-Oportunidades-Prospera, a 
20 años de su creación. Ciudad de México: CONEVAL. 2019. Disponible en: https://www.coneval.org.mx/Evaluacion/IEPSM/Documents/Libro_POP_20.p df Accedido en: 1 sept. 2021.

REYES, Erwin. Operativo Escudo contra coronavirus en Hidalgo. El Sol de Hidalgo. 16 mar. 2020. Disponible en: https://www.elsoldehidalgo.com.mx/local/operativo-escudo-contra-coronav irus-en-hidalgo-4977463.html Accedido en: 17 abr. 2021.

RODRÍGUEZ, Jorge. Vulnerabilidad y grupos vulnerables: un marco de referencia conceptual mirando a los jóvenes. Población y desarrollo, n. 17. Santiago, 2001. https://www.cepal.org/sites/default/files/publication/files/7150/s018659_es.p df Accedido en: 30 ago. 2021.

SECRETARÍA DE SALUD E INSTITUTO NACIONAL DE SALUD PÚBLICA. Fases de la epidemia por COVID-19. 2020. Disponible en: https://www.insp.mx/avisos/5344-fases-de-la-epidemia-por-covid-19.html Accedido en: 30 ago. 2021.

SEDESOL. Lineamientos Progresa Programa de educación, salud y alimentación. DOF. Publicado en: 26 feb. 1999. Disponible en: http://www.dof.gob.mx/nota_detalle.php?codigo=4944430\&fecha=26/02/199 9 Accedido en: 10 mar. 2021.

SEDESOL. Creación del Programa de Desarrollo Humano Oportunidades PDHO, como órgano desconcentrado de la Secretaría de Desarrollo Social. DOF. 6 mar. 2002.2 Disponible en: https://www.dof.gob.mx/nota_to_imagen_fs.php?codnota $=735026 \&$ fecha $=0$ 6/03/2002\&cod_diario=28723 Accedido en: 10 mar. 2021.

SEDESOL. Reglas de Operación del Programa de Desarrollo Humano Oportunidades, para el ejercicio fiscal 2013. DOF. 28 feb. 2013.Disponible en: https://www.dof.gob.mx/nota_detalle.php?codigo=5289882\&fecha=28/02/20 13 Accedido en: 28 mar. 2021.

SEDESOL. Creación de la Coordinación Nacional de PROSPERA Programa de Inclusión Social. DOF. 05 sept. 2014. Disponible en: https://www.dof.gob.mx/nota_to_imagen_fs.php?codnota=5359088\&fecha= 05/09/2014\&cod_diario=260281 Accedido en: 10 mar. 2021.

SEDESOL. Reglas de Operación de PROSPERA Programa de Inclusión Social para el ejercicio fiscal 2016. DOF. 30 dec. 2015. Disponible en: http://www.dof.gob.mx/nota_detalle.php?codigo=5421756\&fecha=30/12/2015 Accedido en: 28 mar. 2021.

SEDESOL. Reglas de Operación de PROSPERA Programa de Inclusión Social para el ejercicio fiscal 2018. DOF. 29 dec. 2017. Disponible en: https://dof.gob.mx/nota_detalle.php?codigo=5509738\&fecha=29/12/2017 Accedido en: 28 mar. 2021 
SEP. Creación de la Coordinación Nacional de Becas para el Bienestar Benito Juárez. DOF. 31 may. 2019. Disponible en: https://www.dof.gob.mx/nota_to_imagen_fs.php?codnota=5561693\&fecha=3 1/05/2019\&cod_diario=281873 Accedido en: 15 mar. 2021.

SEP. Acuerdo por el que se suspenden las clases en las escuelas dependientes de la Secretaría de Educación Pública. DOF. 16 mar. 2020. Disponible en: https://www.dof.gob.mx/nota_detalle.php?codigo=5589479\&fecha=16/03/20 20 Accedido en: 8 abr. 2021.

SSA. Acuerdo por el que se establece una estrategia para la reapertura de las actividades sociales, educativas y económicas, así como un sistema de semáforo para evaluar el riesgo epidemiológico. DOF. Publicado en: 14 may. 2020. Disponible en: https://dof.gob.mx/nota_detalle.php?codigo=5593313\&fecha=14/05/2020 Accedido en: 5 may. 2021.

UNICEF. COVID-19: más del 95 por ciento de niñas, niños y adolescentes está fuera de las escuelas en América Latina y el Caribe. 23 mar. 2020. Disponible en: https://www.unicef.org/lac/comunicados-prensa/covid-19-mas-del-95-por-ci ento-de-ninos-y-ni\%C3\%Blas-esta-fuera-de-las-escuelas Accedido en: 15 abr. 2021. 